\title{
Therapeutic effects of nerve leachate-treated adipose-derived mesenchymal stem cells on rat sciatic nerve injury
}

\author{
YUMEI LIU ${ }^{1 *}$, RUIQI DONG ${ }^{1 *}$, CHUNYAN ZHANG $^{1}$, YUXIANG YANG ${ }^{1}$, YAOLU XU ${ }^{1}$, HAOJIE WANG ${ }^{1}$, \\ MENGYU ZHANG ${ }^{1}$, JIAMIN ZHU ${ }^{1}$, YUQIN WANG ${ }^{1,2}$, YANHONG SUN $^{3}$ and ZIQIANG ZHANG ${ }^{1}$ \\ ${ }^{1}$ College of Animal Science and Technology, Henan University of Science and Technology; \\ ${ }^{2}$ Engineering Research Center for Mutton Sheep Breeding of Henan Province, Luoyang, Henan 471023; \\ ${ }^{3}$ Department of Physiology, Inner Mongolia Medical University, Hohhot, Inner Mongolia 010110, P.R. China
}

Received February 17, 2019; Accepted September 26, 2019

DOI: $10.3892 /$ etm.2019.8203

\begin{abstract}
Peripheral nerve injury (PNI) is a common condition, often resulting from physical nerve injury and trauma. Successful repair of the peripheral nerve is dependent on the regenerative activity of Schwann cells (SCs). Application of SC-like adipose-derived mesenchymal stem cells (ADSCs) may be a suitable cell-based therapy for PNI. In the present study, nerve leachate derived from the rat sciatic nerve was used to induce the differentiation of ADSCs. These cells were placed in an acellular biological scaffold, which was then grafted to a rat sciatic nerve to bridge a $1-\mathrm{cm}$ gap. Sprague-Dawley rats were divided into four groups: Scaffold only, untreated ADSCs + scaffold, nerve leachate-treated ADSCs + scaffold and autograft. Two-months post-transplant, the structure and function of the regenerated nerves and the recovery of the innervated muscles was analyzed. After transplant, there was a significant increase in the average area $(15.86 \% ; \mathrm{P}<0.05)$, density $(23.13 \% ; \mathrm{P}<0.05)$ and thickness $(43.24 \% ; \mathrm{P}<0.05)$ of regenerated nerve fibers in the nerve leachate-treated ADSCs + scaffold group compared with the untreated ADSCs + scaffold group. The nerve conduction velocity in the nerve leachate-treated ADSCs + scaffold and autograft groups was superior to that in the other groups. In the nerve leachate-treated ADSCs + scaffold group, the cross-sectional area of the gastrocnemius increased by $39.28 \%(\mathrm{P}<0.05)$ and the cross-sectional area of collagen fibers decreased by
\end{abstract}

Correspondence to: Dr Ziqiang Zhang, College of Animal Science and Technology, Henan University of Science and Technology, 263 Kaiyuan Avenue, Luolong, Luoyang, Henan 471023, P.R. China E-mail: ziqiangzhang@haust.edu.cn

${ }^{*}$ Contributed equally

Abbreviations: ADSCs, adipose-derived mesenchymal stem cells; MSCs, mesenchymal stem cells; PNI, peripheral nerve injury; SCs, Schwann cells

Key words: peripheral nerve injury, adipose-derived mesenchymal stem cells, Schwann cells
29.87\% $(\mathrm{P}<0.05)$ compared with the ADSCs + scaffold group. Moreover, the therapeutic effect of nerve leachate-treated ADSCs + scaffold on PNI was similar to that of an autograft. These results suggest that nerve leachate-treated ADSCs may promote the repair of PNI.

\section{Introduction}

Peripheral nerve (PN) injury (PNI) is a common condition, occurring in a range of diseases and after a variety of injuries, that can lead to sensory and motor dysfunction or even permanent disability (1). When the peripheral nerve is severely damaged, a series of metamorphoses and phagocytic processes, such as axonal necrosis and degradation of the myelin sheath, occur in distal nerves, in a process referred to as Wallerian degeneration (2). Structural and sensory problems, such as innervated muscle atrophy and localized sensory loss of innervation, also occur after PNI (3). Peripheral nerve (PN) regeneration is a complex process associated with the elimination of inflammation, release of neurotrophic factors, axonal growth and neuron survival (3). Successful peripheral nerve regeneration after injury depends on dynamic changes in Schwann cells (SCs) $(4,5)$. After PNI, SCs begin to divide and proliferate, forming a cord-like channel, known as a Büngner band, surrounded by basal lamina (6). SCs synthesize and release neurotrophic and cell adhesion factors that stimulate the regeneration of myelin and axons and provide the necessary attachment surfaces for regenerating axons $(7,8)$. However, the regeneration of injured nerves is slow, due to the complexity of the repair process, and prolonged denervation of the proximal nerve and neural mismatch may result in irreversible atrophy of the associated organ $(9,10)$. For this reason, PN repair remains a considerable clinical challenge.

Currently the gold standard treatment for PNI is autologous nerve transplantation, however, this treatment has a number of limitations, such as shortage of donor sources, the need for an extra incision, nerve matching problems, and possible loss of neurological function at the donor site $(11,12)$.

Due to advances in tissue engineering, tissue-engineered nerve scaffolds provide a promising new strategy for the treatment of PNI (13). This method of tissue engineered nerve grafting involves the use of seed cells that are cultured 
in vitro and anchored to a natural or synthetic biocompatible conduit to form a composite nerve scaffold. This scaffold can be implanted in vivo to bridge gaps between injured nerves. Cells attached to scaffolds have shown some positive effects in nerve regeneration (14). However, SCs, which are the central factors required for $\mathrm{PN}$ regeneration, are difficult to obtain and expand to sufficient numbers. Tissue availability is limited, due to problems with morbidity of the donor site and the risk of sacrifice of one or more normal nerves leading to a loss of sensation $(15,16)$. Furthermore, SCs are terminal cells (17). Although techniques to increase the acquired number and survival rates of SCs in vitro have been developed $(18,19)$, further studies are required to make these a viable option. In the last decade, the field of regenerative medicine has been focused on stem cells, which may be useful in PNI treatment as they can be differentiated into Schwann (SC)-like cells through physical and chemical induction methods $(20,21)$.

Adipose-derived mesenchymal stem cells (ADSCs) are adult mesenchymal stem cells (MSCs) with self-renewal ability and multidirectional differentiation potential (20). Compared with bone marrow-derived MSCs (BMSCs), ADSCs have various advantages, such as the ease of their extraction, their abundance in adipose tissues and their rapid rate of proliferation (22). A number of studies have confirmed that PNI can be alleviated after ADSC transplantation into nerve injury sites $(23,24)$. A number of studies have previously demonstrated that ADSC transplantation has a regenerative effect on sciatic nerve injury in a rat model $(25,26)$. These studies found that only a small number of ADSCs survived in vivo and that the rate of ADSC differentiation into SC-like cells at the site of injury was low. Therefore, the success of PNI treatment with undifferentiated ADSCs is not guaranteed (27). Several investigators have induced ADSC differentiation into SC-like cells in vitro and then transplanted these cells in vivo, confirming that SC-like cells differentiated from ADSCs can promote peripheral nerve regeneration $(28,29)$. However, these differentiation procedures have a number of disadvantages, including cumbersome protocols, high cost and low differentiation rate $(30,31)$. The results of previous studies suggested that SCs secrete neurotrophic factors, which are released by injured nerve endings during PNI, that can promote axon growth and protect neurons (32). Based on these findings, injured nerve endings were placed in culture medium to generate a neurotrophic substance-rich nerve leachate (33-35). A previous study showed that nerve leachate induced the in vitro differentiation of ADSCs into SC-like cells, which expressed specific SC markers (36). This differentiation method is convenient, economical and simple. However, whether nerve leachate-treated ADSCs can influence PNI repair has yet to be confirmed in an in vivo study. In the present study, a 1-cm length of sciatic nerve was removed, in order to establish a model of PNI, and nerve leachate-differentiated ADSCs on acellular nerve scaffolds were transplanted to bridge this defect. The repair effects of nerve leachate-treated ADSCs on rat sciatic nerve injury were determined through investigation of indicators including nerve regeneration and gastrocnemius recovery.

\section{Materials and methods}

Animals. Two groups of male Sprague-Dawley (S-D) rats (6; age, 3-weeks; weight, 45-55 g; and 21; age, 8-weeks; weight range, 180-210 g) were obtained from the Experimental Animal Center of Zhengzhou University Medical College (Zhengzhou, China). Three-week-old rats were used to isolate and culture ADSCs, whereas eight-week-old rats were used for in vivo experiments. The animals were housed at $20-26^{\circ} \mathrm{C}$ with 50-60\% humidity, under a 12-h light/dark cycle and had free access to water and food. Experiments were designed to minimize animal suffering and reduce the number of experimental animals used. All animal care and experimental protocols were conducted in accordance with university policies on the use and care of animals and were approved by the Institutional Animal Experiment Committee of Henan University of Science and Technology (Henan, China).

Isolation and culture of rat ADSCs. After the sacrifice of 6 3 -week old rats, adipose tissue was isolated from the inguinal region and minced under aseptic conditions. This isolated adipose tissue was digested at $37^{\circ} \mathrm{C}$ for $60 \mathrm{~min}$ with $0.1 \%$ collagenase type I (Gibco; Thermo Fisher Scientific, Inc.). The resulting suspension was centrifuged at $175 \mathrm{x} \mathrm{g}(1,000 \mathrm{rpm})$ for $10 \mathrm{~min}$ at room temperature to separate the ADSCs from the stromal vascular fraction (36). The precipitated cells were resuspended and cultured at $37^{\circ} \mathrm{C}$ in $5 \% \mathrm{CO}_{2}$ in Dulbecco's modified Eagle's medium (DMEM; Gibco; Thermo Fisher Scientific, Inc.) supplemented with $10 \%$ fetal bovine serum (FBS; Gibco; Thermo Fisher Scientific, Inc.). Cells that did not adhere to tissue culture plastic after $24 \mathrm{~h}$ were removed when the medium was replaced. Rat ADSCs were passaged 3-5 times before use.

Verification of ADSC multi-lineage differentiation and expression of specific surface markers. Using the method reported by $\mathrm{Fu}$ et al (16), passage 3 rat ADSCs were seeded into six-well plates at a density of $5 \times 10^{5}$ cells $/ \mathrm{ml}$. When the ADSCs were $80 \%$ confluent, normal medium was replaced with medium to induce their differentiation. ADSCs were cultured for 14 days in adipogenic differentiation medium (DMEM supplemented with $10 \%$ FBS, $0.5 \mathrm{mM}$ isobutylmethylxanthine, $1 \mu \mathrm{M}$ dexamethasone, $10 \mu \mathrm{M}$ insulin and $200 \mu \mathrm{M}$ indomethacin; Sigma-Aldrich; Merck KGaA) (36). The cells were fixed by $4 \%$ paraformaldehyde for $30 \mathrm{~min}$ at room temperature, then washed with PBS for two times and the lipid droplets present in the cells were stained using oil red $\mathrm{O}$ for $30 \mathrm{~min}$ at room temperature (Sigma-Aldrich; Merck $\mathrm{KGaA}$ ). Rat ADSCs were cultured for 21 days in osteogenic differentiation medium (DMEM supplemented with $10 \% \mathrm{FBS}$, $100 \mathrm{nM}$ dexamethasone, $50 \mu \mathrm{M}$ ascorbate-2-phosphate, and $10 \mathrm{mM} \beta$-glycerophosphate) (37). The cells were fixed by $4 \%$ paraformaldehyde for $30 \mathrm{~min}$ at room temperature, then washed two times with PBS before intracellular calcium deposition in the cells was assessed by von Kossa staining for $30 \mathrm{~min}$ at room temperature (all reagents are from Sigma-Aldrich; Merck KGaA).

The immunophenotype of ADSCs was determined by flow cytometry. Passage 3 rat ADSCs were washed with PBS and then the cells were fixed by $4 \%$ paraformaldehyde for $30 \mathrm{~min}$ at room temperature. The fixed cells were incubated with FITC-coupled antibodies against rat CD31 (1:200; cat. no. MCA1334G), CD44 (1:200; cat. no. MCA643), CD45 (1:200; cat. no. MCA43) and CD90 (1:200; cat. no. MCA47; 
Bio-Rad Laboratories, Inc.) in the dark at room temperature for 30 min and analyzed by a flow cytometer (FACSCalibur ${ }^{\mathrm{TM}}$; Becton, Dickinson and Company) using the FlowJo Software (version 7.6.1; FlowJo LLC).

Preparation of the nerve leachate. According to a previously reported method (36), rat sciatic nerves were removed from 9 male 8-weeks old rats under aseptic conditions, and cut into $2-\mathrm{cm}$ segments. After washing the nerve segments three times with PBS containing $0.2 \%$ penicillin-streptomycin (Gibco; Thermo Fisher Scientific, Inc.), they were transferred into $100 \mathrm{ml} \mathrm{DMEM}$ medium containing 10\% FBS, following which the medium containing the sciatic nerve was stored at $4^{\circ} \mathrm{C}$ for 3 days. The culture medium was removed on the third day and filtered with a $0.22 \mu \mathrm{m}$ microporous membrane filter. The nerve leachate was prepared prior to this study.

Induction of rat ADSCs into SC-like cells. Passage 3 ADSCs were used in all experiments. Briefly, culture medium was removed from sub-confluent cultures of ADSCs and replaced with nerve leachate medium, which was in turn refreshed once every 1-2 days. Cells were then incubated for 5 days at $37^{\circ} \mathrm{C}$ under $5 \% \mathrm{CO}_{2}$. Morphological changes were observed using a light microscope (Olympus Corporation) after ADSCs were inducted for $96 \mathrm{~h}$ using the nerve leachate medium.

Preparation of the tissue-engineered nerve scaffold. Acellular nerve scaffolds were prepared using the method of Zhang et al $(37,38)$ from five 8 -week-old male rats, which were prepared prior to this study. Sciatic nerve segments isolated from rats were placed in distilled water and underwent 3 cycles of freezing at $-80^{\circ} \mathrm{C}$ for $60 \mathrm{~min}$ and thawing at room temperature for $20 \mathrm{~min}$ per cycle. The nerve segments were digested with $40 \mathrm{U} / \mathrm{ml}$ DNase (Sigma-Aldrich; Merck KGaA) and RNase (Sigma-Aldrich, Merck $\mathrm{KGaA}$ ) at $37^{\circ} \mathrm{C}$ for $2 \mathrm{~h}$. They were then immersed in $4 \%$ sodium hydroxide solution at $4^{\circ} \mathrm{C}$ for $24 \mathrm{~h}$. After washing with $\mathrm{PBS}$, the nerve segments were lyophilized, sterilized and stored at $4^{\circ} \mathrm{C}$.

Suspensions of untreated ADSCs and nerve leachate-treated ADSCs containing $5 \times 10^{7}$ cells were prepared and placed in centrifuge tubes. A total of $2 \mathrm{ml}$ collagen (Sigma-Aldrich; Merck $\mathrm{KGaA}$ ) was added to the centrifuge tubes at $4^{\circ} \mathrm{C}$ and the suspensions were mixed rapidly. Collagen with or without cells was slowly injected into the end of the acellular nerve scaffolds. The composite nerve scaffolds that contained cells and collagen were then transferred to a Petri dish, incubated at $37^{\circ} \mathrm{C}$ under $5 \% \mathrm{CO}_{2}$ in $5 \mathrm{ml} \mathrm{DMEM}$ containing $10 \% \mathrm{FBS}$ for 4 days.

Surgical transplantation. The S-D rats were randomly divided into the following four groups ( $n=3$ per group): Scaffold only, untreated ADSCs + scaffold, nerve leachate-treated ADSCs + scaffold, and autograft. The rats were anesthetized with $1 \%$ pentobarbital sodium $(50 \mathrm{mg} / \mathrm{kg})$ by intraperitoneal injection. The fur of the right hind limb was shaved and the skin was sterilized. A skin incision was made along the femoral axis of the right hind leg, and the thigh muscles were separated. The sciatic nerve was exposed through the thigh muscle interval, and a 1-cm nerve segment was removed (39).
An acellular nerve scaffold, an acellular nerve scaffold combined with ADSCs, an acellular scaffold combined with nerve leachate-treated ADSCs or an autograft nerve were transplanted into this $1-\mathrm{cm}$ segment to bridge the nerve gap. The muscle layers and skin were sutured with 4-0 sutures. After surgery, rat mental state, physical activity, diet, wound healing and foot ulcer formation were checked regularly. After two months, all rats were sacrificed by cervical dislocation following intraperitoneal injection with $3 \%$ sodium pentobarbital $(50 \mathrm{mg} / \mathrm{kg})$.

Electrophysiological assessment. Electrophysiological analysis was performed on the rats at 2 months post-surgery. Under anesthesia, the bilateral sciatic nerves of the rats were exposed. Electrical stimuli were then applied to the proximal portion of the nerve trunk, and the nerve action potential (NAP) was measured at a distance of $10 \mathrm{~mm}$ from the electrical stimulation site. The nerve conduction velocity (NCV) was analyzed as a function of distance and time.

Histological assessment. Two months after the surgery, the rats were sacrificed by cervical dislocation under anaesthesia by intraperitoneal injection with $3 \%$ sodium pentobarbital $(50 \mathrm{mg} / \mathrm{kg})$. The rat sciatic nerve was re-exposed and the regenerated nerve was harvested. The nerve segment was fixed with $2 \%$ glutaraldehyde at $4^{\circ} \mathrm{C}$ for $1 \mathrm{~h}$ and post-fixed with $1 \%$ osmium tetroxide at $4^{\circ} \mathrm{C}$ for $1 \mathrm{~h}$ before it was embedded in epoxy resin. The embedded samples were cut into thin sections $(0.5-1.0 \mu \mathrm{m})$. These thin sections were stained with $1 \%$ toluidine blue at $60^{\circ} \mathrm{C}$ for $5 \mathrm{~min}$ and the density of myelinated nerve fibers was observed under a light microscope (Olympus Corporation). The nerve segments were fixed in $2 \%(\mathrm{v} / \mathrm{v})$ glutaraldehyde in a $0.1-\mathrm{M}$ phosphate buffer (pH 7.2) at $4^{\circ} \mathrm{C}$ for $\geq 2 \mathrm{~h}$ and washed three times with PBS at room temperature. The samples were then post-fixed with $1 \%(\mathrm{w} / \mathrm{v})$ osmium tetroxide at $4^{\circ} \mathrm{C}$ for $2 \mathrm{~h}$ followed by a further three-time PBS wash at room temperature. The samples were dehydrated by using an alcohol gradient, replaced with acetone and embedded in epoxy resin for $2 \mathrm{~h}$ at $45^{\circ} \mathrm{C}$ and polymerized at $60^{\circ} \mathrm{C}$ for $48 \mathrm{~h}$. Ultrathin sections $(50 \mathrm{~nm})$ were then lifted onto formvar-coated grids, post-stained with lead citrate for $15 \mathrm{~min}$ and uranyl acetate for $40 \mathrm{~min}$ at room temperature, and observed by transmission electron microscopy (TEM; Hitachi, Ltd.). The total number of myelinated nerve fibers and myelin thickness and area, specifically the TEM data, were analyzed using HPIAS-1000-type high-resolution color graphic pathological analysis system (HPIAS-1000-type; Tongji Medical University, Wuhan, China).

Two months after surgery, the gastrocnemius tissue was removed and embedded in paraffin and cut into thin sections $(5-10 \mu \mathrm{m})$. The sections were stained using Masson's trichrome (40). The results were observed under a light microscope and the cross-sectional area of the gastrocnemius muscle was measured.

Statistical analysis. The data were analyzed with SPSS 18.0 software (SPSS, Inc.). Each experiment was performed at least three times. All quantitative data are presented as the mean \pm standard deviation. Differences between the groups were compared by one-way analysis of variance and Duncan's 

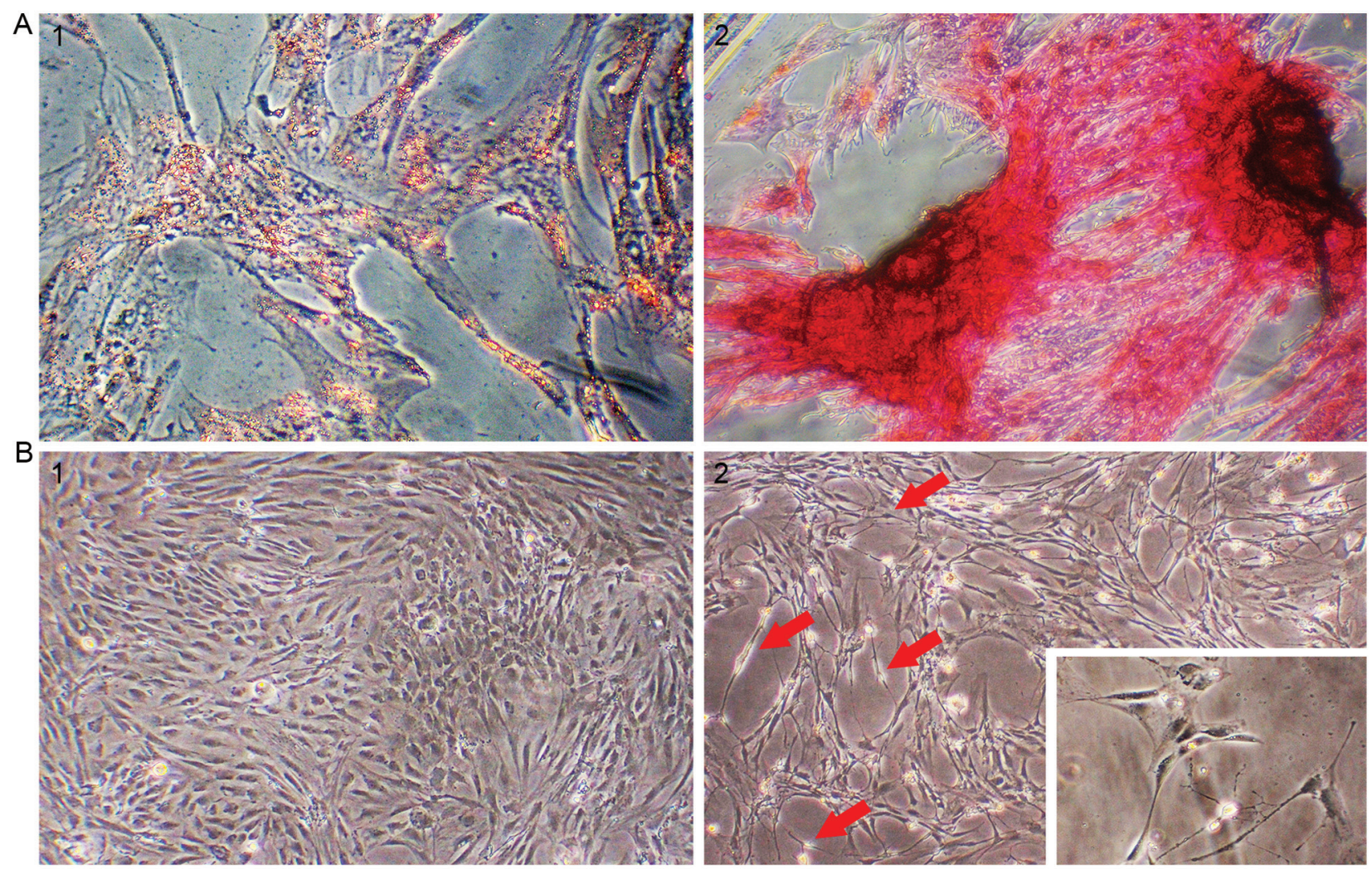

C
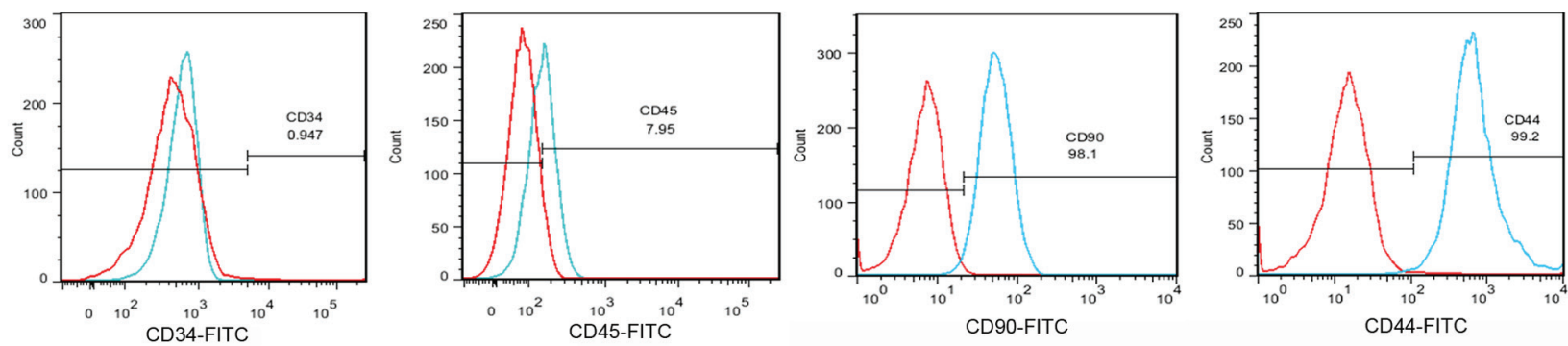

Figure 1. Characterization of ADSCs and differentiation of ADSCs into Schwann-like cells. (A-1) Cells were observed to contain red lipid droplets after staining with oil red O. (A-2) Cells showed black calcium nodule deposition after staining with von Kossa stain (magnification, x400). (B-1) The morphology of passage 3 undifferentiated ADSCs. (B-2) The morphology of ADSCs treated with nerve leachate for $96 \mathrm{~h}$ (magnification, x200). (C) Flow cytometry analysis indicated that the isolated cells expressed ADSCs surface markers CD44/CD90 and did not express CD34/CD45. The red line indicates the isotype control and the blue line indicates ADSCs. ADSC, adipose-derived mesenchymal stem cell.

multiple comparison tests. $\mathrm{P}<0.05$ was considered statistically significant.

\section{Results}

Morphology and multidirectional differentiation of rat ADSCs. Passage 3 rat ADSCs exhibited a regular spindle shape (Fig. 1B-1). ADSCs were treated with induction medium to investigate whether cells isolated from adipose tissue have the multidirectional differentiation ability of MSCs. After rat ADSCs were incubated in adipogenic differentiation medium for 2 weeks, lipid droplets in the cells accounted for approximately $80 \%$ of the cell volume, and adipocyte differentiation was confirmed by lipid droplet staining with oil red $\mathrm{O}$ (Fig. 1A-1). After 21 days of culture in osteogenic differentiation medium, osteogenic differentiation was confirmed by the presence of calcium deposits stained with von Kossa staining (Fig. 1A-2). Flow cytometry analysis of passage 3 rat ADSCs showed that the cultured ADSCs were positive for CD44 and CD90, and negative for CD34 and CD45 (Fig. 1C).

Nerve leachate induces morphological changes in ADSCs. The cells began to change the cytoplasm from a flat to contractile shape after treating with nerve leachate for $24 \mathrm{~h}$. After $48 \mathrm{~h}$, the cells showed a mixture of morphologies, with the cells changing from long fusiform to a bi- or tri-polar elongated spindle shapes. After the cells were treated for $96 \mathrm{~h}$, the cells had morphologies similar to those of SCs, namely cytoplasmic extension and large nuclei (Fig. 1B-1 and B-2).

Rat health observations. The rats in each group had a good mental state after surgery and no infections occurred in the wounds. One month after surgery, the incisions and ulcers of the rats in the nerve leachate-treated ADSCs + scaffold and autograft groups recovered better than those of the rats in the scaffold only group. Two months after surgery, the rats in the 
A
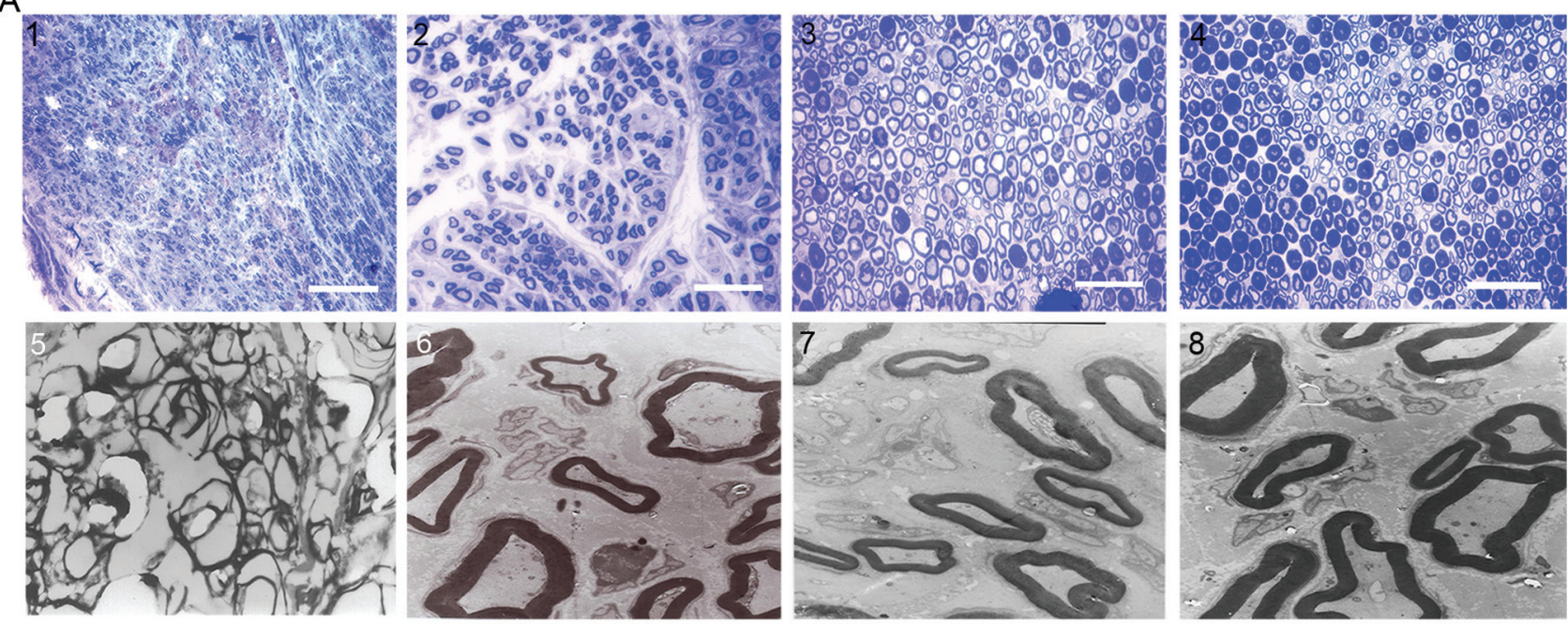

B
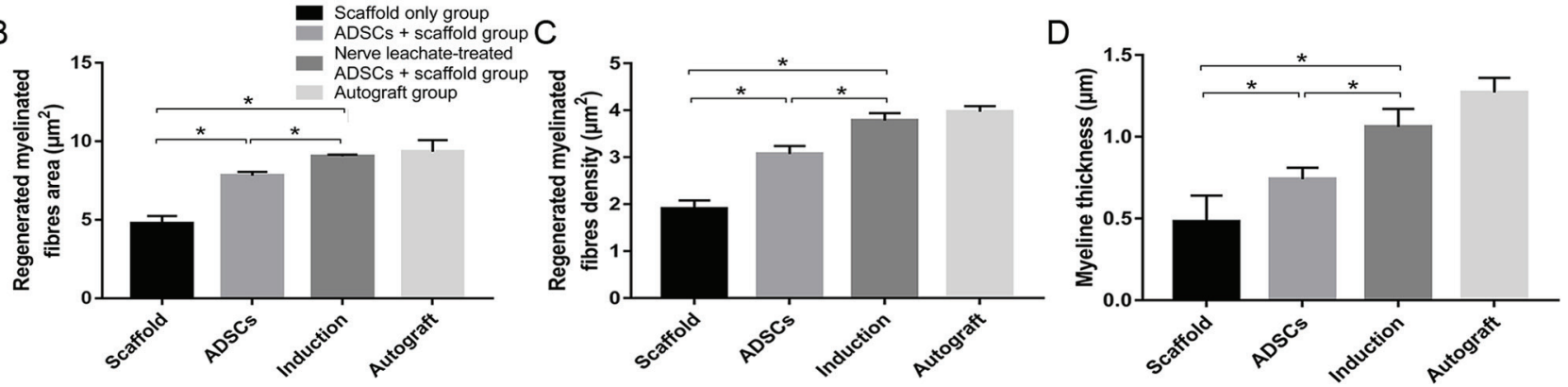

Figure 2. Histological assessment of regenerated nerves. Regenerated nerves stained with toluidine blue in the (A-1) scaffold only group, (A-2) untreated ADSCs + scaffold group (A-3) nerve leachate-treated ADSCs + scaffold group and (A-4) autograft group (magnification, $\mathrm{x} 400$ ). TEM results showing regenerated nerves in the (A-5) scaffold only group, (A-6) untreated ADSCs + scaffold group (A-7) nerve leachate-treated ADSCs + scaffold group and (A-8) autograft group (magnification, $\mathrm{x} 4,000$ ). (B) The regenerated myelinated fiber area, (C) regenerated myelinated fiber density and (D) myelin thickness were analyzed in each group. Scale bar, $20 \mu \mathrm{m}$. The data are presented as the mean \pm SD. ${ }^{*} \mathrm{P}<0.05$. ADSC, adipose-derived mesenchymal stem cell; TEM, transmission electron microscopy.

nerve leachate-treated ADSCs + scaffold and autograft groups were able to walk normally. When all rats were sacrificed to re-expose the sciatic nerve, the damaged nerves had regained connectivity in all groups.

Histological assessment of regenerated nerves. TEM and toluidine blue staining results indicated that a smaller number of regenerated nerve fibers formed in the scaffold only group compared with the untreated ADSCs and the nerve leachate-treated ADSCs + scaffold group, which were irregularly arranged (Fig. 2A). Several regenerated nerve fibers were observed in the ADSCs + scaffold group, but they were less abundant than those in the nerve leachate-treated ADSCs + scaffold and autograft groups. The largest numbers of regenerated nerve fibers, which were dispersed in clusters with thick myelin sheaths, were observed in the nerve leachate-treated ADSCs + scaffold and autograft groups. Based on the results of image analysis, the average area of the nerve fibers in the nerve leachate-treated ADSCs + scaffold group (15.86\%), the nerve fiber density (23.13\%), and the nerve fiber thickness (43.24\%) increased significantly compared with the untreated ADSCs + scaffold group (Fig. 2B-D).

Electrophysiological assessment of regenerated nerves. Electrophysiological examination of the regenerated nerves in each group at 2 months after the operation showed that the recovery rates of NAP and NCV were significantly reduced in the scaffold only group compared with those in the untreated ADSCs and the nerve leachate-treated ADSCs + scaffold group. In addition, they were higher in the untreated ADSCs + scaffold group compare with those in the scaffold only group. The rates of NAP and NCV in the nerve leachate-treated ADSCs + scaffold group were significantly greater than those in the untreated ADSCs + scaffold and scaffold only groups. There was no significant difference between the nerve leachate-treated ADSCs + scaffold group and the autograft groups (Fig. 3A and B).

Assessment of the gastrocnemius. Masson's trichrome stained hyperplastic collagen fibers green in muscle bundles (Fig. 4A). The number of hyperplastic fibers in the scaffold only group was markedly increased compared with the untreated ADSCs and the nerve leachate-treated ADSCs + scaffold group and a lower number of hyperplastic fibers were observed in the untreated ADSCs + scaffold group compared with the scaffold only group. Hyperplastic fibers were not clearly observed in the nerve leachate-treated ADSCs and autograft groups. The results of the image analysis system indicated that the cross-sectional area of the gastrocnemius and the area of collagen fibers in the scaffold group were 115.11 and 

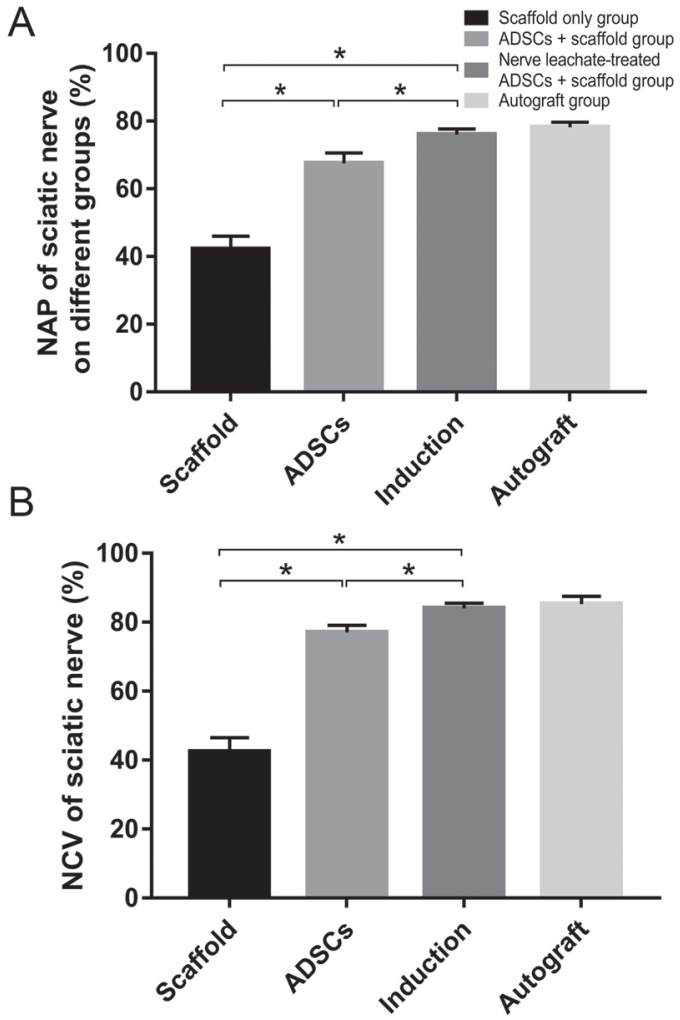

Figure 3. Electrophysiological assessment. (A) Electrical stimuli were applied to the proximal portion of the nerve trunk, and the amplitude of the NAP was recorded. (B) NCV was analyzed as a function of passing distance and time. The data are presented as the mean $\pm \mathrm{SD} .{ }^{*} \mathrm{P}<0.05$. ADSC, adipose-derived mesenchymal stem cell; NAP, nerve action potential; NCV, nerve conduction velocity.

$66.56 \mu \mathrm{m}^{2}$, respectively. Compared with the scaffold only group, the cross-sectional area of gastrocnemius increased by $66.78 \%$ and the area of collagen fibers decreased by $73.65 \%$ in the untreated ADSCs + scaffold group (Fig. 4B and C). Compared with the untreated ADSCs + scaffold group, the cross-sectional area of gastrocnemius, and the area of collagen fibers in the nerve leachate-treated ADSC + scaffold group increased by $39.28 \%$ and decreased by $29.87 \%$, respectively (Fig. 4B and C). There were no significant differences between the autograft and the nerve leachate-treated ADSCs + scaffold groups.

\section{Discussion}

In contrast to the central nervous system, the peripheral nervous system has excellent regeneration and self-repair abilities (41), particularly in favorable microenvironments (42). When PNI occurs, SCs undergo morphological changes to guide axonal regeneration, and trophic factors and adhesion factors released by SCs promote nerve repair $(43,44)$. However, the injury site does not provide a suitable microenvironment, hindering the self-repair process of long-gap nerve defects (33).

The rapid development of tissue engineering technology provides opportunities for the repair of PNI. Composite nerve grafts not only guide axonal regeneration, but also create a favorable microenvironment to ensure the effective treatment of nerve endings with various nutrient factors (14). A mature tissue-engineered nerve scaffold, which can create a better regenerative microenvironment following PNI, should have the following three components: A biological scaffold, seed cells and nutrient factors. Biological scaffolds and seed cells play a crucial role in tissue engineering. MSCs have been investigated as key seed cells in tissue-engineered scaffold transplantation due to their multidirectional differentiation and self-renewal properties (45). BMSCs were the first discovered MSCs and have been shown to differentiate into SC-like cells under certain conditions $(46,47)$. ADSCs have similar properties to BMSCs and are easier to acquire (48). Previous studies have indicated that ADSCs express CD29, CD44, CD90 and CD105 but are negative for the hematopoietic cell lineage markers CD34, CD45, CD31 and CD106 (49,50). In the present study, flow cytometry analysis showed that the isolated cells were positive for CD44/CD90 but not CD34/CD45, which was consistent with the phenotypic characteristics of ADSCs. A number of studies have shown that ADSCs can be differentiated into SC-like cells using various methods, such as chemical $(51,52)$ and co-culture methods $(53)$. In both of these methods ADSCs differentiation into SC-like cells is induced by neurotrophic factors, including nerve growth factor and brain-derived neurotrophic factor. These are added to differentiation medium or directly secreted by SCs (54). Fundamentally, neurotrophic factors are the key to promoting the differentiation of stem cells into neuron-like cells (55). In a previous study, the differentiation of ADSCs into SC-like cells was induced in vitro by nerve leachate (36).

In the present study, the regeneration of the sciatic nerve was assessed after a 2-month healing period. This length of time was chosen as compared with crushing injuries, transection injuries require more than 3-4 weeks for recovery (56). In addition, studies have shown that stem cells are still present and survive at the site of injury after transplantation of composite nerve conduits at 6 weeks after PNI (57). The present study used electrophysiological assessment to indicate the continuity of the regenerated nerve. The NAP and NCV of the sciatic nerve reached 76.08 and $84.07 \%$, respectively, in the nerve leachate-treated ADSCs + scaffold group. These results suggested that a $1-\mathrm{cm}$ injury to the sciatic nerve was repaired by regenerated nerve and that the conduction function of the nerve in the nerve leachate treated ADSC + scaffold group was superior to that in the untreated ADSC + scaffold group. Morphological analysis is commonly used to assess the quality of a regenerative nerve (58). In the present study, the results of TEM suggested that the number of nerve fibers was greater and that the myelin sheath was thicker in the nerve leachate-treated ADSCs + scaffold group. The same result was observed with toluidine blue staining. When peripheral nerves are damaged, muscle atrophy occurs in the gastrocnemius muscle (10), which is controlled by the sciatic nerve. Thus, the recovery of peripheral nerves was evaluated by observing pathological changes in the gastrocnemius muscle stained with Masson's trichrome (59). No obvious pathological changes were seen in the gastrocnemius muscle tissue in the nerve leachate-treated ADSCs + scaffold group. Moreover, the cross-sectional area of the gastrocnemius muscle in the nerve leachate-treated ADSCs + scaffold group was larger compared with the untreated ADSCs and the scaffold only group, indicating that the degree of gastrocnemius atrophy in the rats from this group was slight. The gastrocnemius muscles in the 

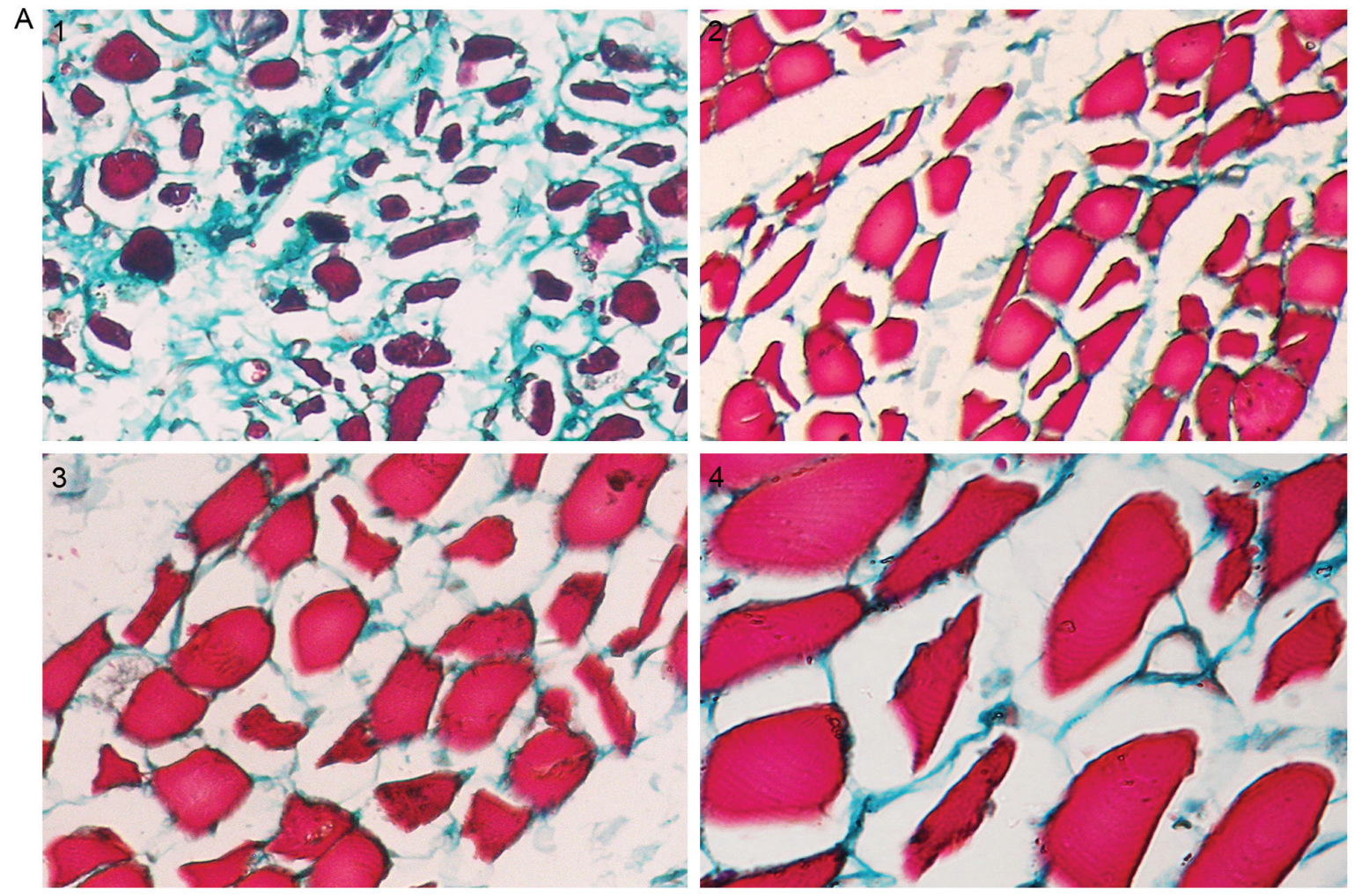

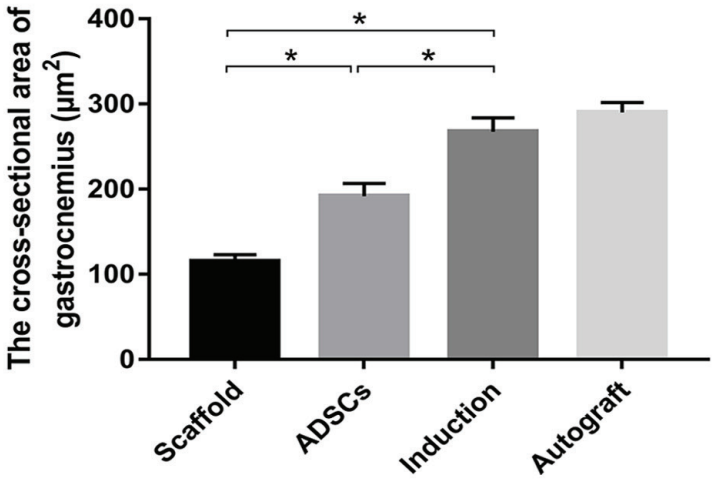

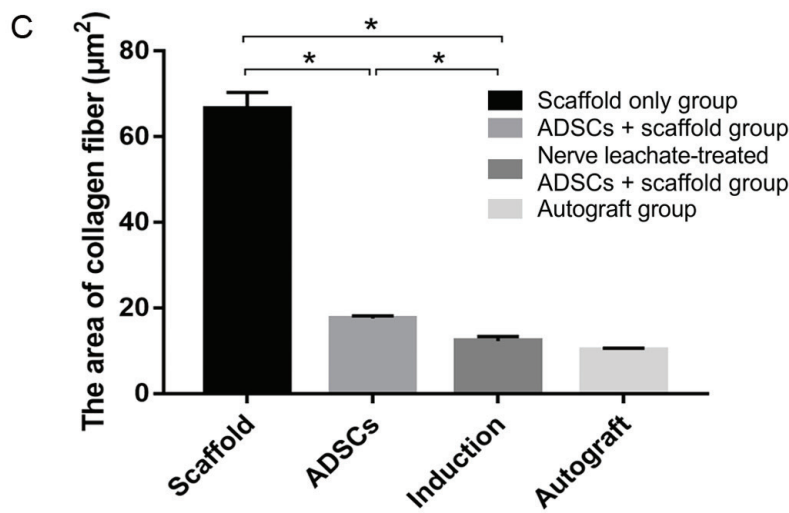

Figure 4. Assessment of the gastrocnemius. The gastrocnemius from (A-1) the scaffold only group, (A-2) untreated ADSCs + scaffold group, (A-3) nerve leachate-treated ADSCs + scaffold group and (A-4) autograft group were stained using Masson's trichrome. (B) Cross-sectional area of the gastrocnemius. (C) Area of the collagen fibers in the gastrocnemius. Scale bar, $20 \mu \mathrm{m}$. Data are presented as the mean $\pm \mathrm{SD}$. "P $<0.05$. ADSC, adipose-derived mesenchymal stem cell.

scaffold only group had a large area of collagen fibers, and muscle atrophy in the scaffold only group was more obvious compared the nerve leachate-treated ADSCs + scaffold and the untreated ADSCs group. This demonstrated that somatic function dominated by the sciatic nerve recovered effectively in the nerve leachate-treated ADSCs + scaffold group.

Autograft was chosen as a positive control because autografting is the gold standard therapeutic method for peripheral nerve repair in the clinic (59). The results of the present study indicated that the recovery level in the nerve leachate-treated ADSCs + scaffold group was similar to that in the autograft group. As expected, the composite nerve scaffolds containing nerve leachate-treated ADSCs resulted in better functional and structural recovery during the treatment of PNI than the scaffold only and the untreated ADSCs + scaffold group. ADSCs treated with nerve leachate may have therapeutic potential for PNI.
Previous studies have suggested that SCs undergo dedifferentiation, and their secretion of factors, such as neurotrophic factors, brain-derived neurotrophic factor $(60,61)$, neuronal growth factor (62) and cell adhesion factors, increases after PNI. These factors regulate the structure and function of the nervous system and play a key role in the repair of PNI (63). In the present study, rat sciatic nerves were cut and used to prepare a nerve leachate. Results suggested that ADSCs induced with this leachate may play an effective therapeutic role in PNI. This leachate may contain various neurotrophic factors released by the nerve ends that induce the differentiation of ADSCs. In previous work, nerve leachate was prepared from the sciatic nerves of animals, such as rabbits, dogs and frogs, and was also shown to induce the differentiation of ADSCs into SC-like cells (unpublished data). In addition, research revealed that nerve leachate from the sciatic nerves of cattle induced the neural differentiation of 
rat $\mathrm{PC} 12$ cells (60). The above results indicated that there is no species limitation for the source of nerve leachate that can be used to induce the differentiation of ADSCs. In addition, the nerves from different animals can be obtained for the preparation of nerve leachate from animal feed houses and slaughterhouses, such as barns and pig farms. During the preparation of nerve leachate, in order to ensure the consistent quality, animals of the same species with similar body weight were selected, where the sciatic nerve segment of the same length and diameter were taken and the preparation method of the nerve leachate was also kept consistent. However, although it was demonstrated in the present study to exert positive effects, differences in the nerve leachate from different species were not determined. The composition of the nerve is complex due to the presence of a variety of positive nerve factors $(32,60,61)$. Any future research should focus on the study of characterizing and comparing the components of the nerve leachate from different species. Co-culture and chemical induction methods to induce the differentiation of ADSCs into SC-like cells exist, but both methods have limitations. For example, co-culture is cumbersome, and some of the neurotrophic factors for chemical induction are expensive. In the present study, preparation of nerve leachate was simple and used abundant biological materials.

\section{Acknowledgements}

No applicable.

\section{Funding}

This work was supported by grants from the National Natural Science Foundation of China (grant nos. U1504325 and 31101779) and the Undergraduate Training Programs in Henan University of Science and Technology (grant no. 2018380).

\section{Availability of data and materials}

The datasets used and/or analyzed during the present study are available from the corresponding author on reasonable request.

\section{Authors' contributions}

YL, RD and ZZ conceived and designed the study. RD, CZ, YY, YX, HW, MZ, JZ, YW and YS performed the experiments. YL and RD wrote the manuscript. The manuscript was reviewed and edited by ZZ. All authors have read and approved this manuscript.

\section{Ethics approval and consent to participate}

All animal care and experimental protocols were conducted according to the University Policies on the Use and Care of Animals and were approved by the Institutional Animal Experiment Committee of Henan University of Science and Technology (Henan, China).

\section{Patient consent for publication}

Not applicable.

\section{Competing interests}

The authors declare that they have no competing interests.

\section{References}

1. Campbell WW: Evaluation and management of peripheral nerve injury. Clin Neurophysiol 119: 1951-1965, 2008.

2. Conforti L, Gilley J and Coleman MP: Wallerian degeneration: An emerging axon death pathway linking injury and disease. Nat Rev Neurosci 15: 394-409, 2014.

3. Faroni A, Mobasseri SA, Kingham PJ and Reid AJ: Peripheral nerve regeneration: Experimental strategies and future perspectives. Adv Drug Deliv Rev 82-83: 160-167, 2015.

4. Wang X, Luo E, Li Y and Hu J: Schwann-like mesenchymal stem cells within vein graft facilitate facial nerve regeneration and remyelination. Brain Res 1383: 71-80, 2011.

5. Dubey N, Letourneau PC and Tranquillo RT: Guided neurite elongation and schwann cell invasion into magnetically aligned collagen in simulated peripheral nerve regeneration. Exp Neurol 158: 338-350, 1999.

6. Chen ZL, Yu WM and Strickland S: Peripheral regeneration. Annu Rev Neurosci 30: 209-233, 2007.

7. Mirsky R, Jessen KR, Brennan A, Parkinson D, Dong Z, Meier C, Parmantier E and Lawson D: Schwann cells as regulators of nerve development. J Physiol Paris 96: 17-24, 2002.

8. Matsuyama T, Mackay M and Midha R: Peripheral nerve repair and grafting techniques: A review. Neurol Med Chir (Tokeyo) 40: 187-199, 2000.

9. Griffin JW, Hogan MV, Chhabra AB and Deal DN: Peripheral nerve repair and reconstruction. J Bone Joint Surg Am 95: 2144-2151, 2013.

10. Brull R,Hadzic A, Reina MA and Barrington MJ: Pathophysiology and etiology of nerve injury following peripheral nerve blockade. Reg Anesth Pain Med 40: 479-490, 2015.

11. Scheib J and Hoke A: Advances in peripheral nerve regeneration. Nat Rev Neurol 9: 668-676, 2013.

12. Trehan SK, Model Z and Lee SK: Nerve repair and nerve grafting. Hand Clin 32: 119-125, 2016.

13. Brooks DN, Weber RV, Chao JD, Rinker BD, Zoldos J, Robichaux MR, Ruggeri SB, Anderson KA, Bonatz EE, Wisotsky SM, et al: Processed nerve allografts for peripheral nerve reconstruction: A multicenter study of utilization and outcomes in sensory, mixed, and motor nerve reconstructions. Microsurgery 32: 1-14, 2012.

14. Sarker MD, Naghieh S, McInnes AD, Schreyer DJ and Chen X: Regeneration of peripheral nerves by nerve guidance conduits: Influence of design, biopolymers, cells, growth factors, and physical stimuli. Prog Neurobiol 171: 125-150, 2018.

15. Hayashi A, Moradzadeh A, Tong A, Wei C, Tuffaha SH, Hunter DA, Tung TH, Parsadanian A, Mackinnon SE and Myckatyn TM: Treatment modality affects allograft-derived Schwann cell phenotype and myelinating capacity. Exp Neurol 212: 324-336, 2008.

16. Fu X, Tong Z, Li Q, Niu Q, Zhang Z, Tong X, Tong L and Zhang X: Induction of adipose-derived stem cells into Schwann-like cells and observation of Schwann-like cell proliferation. Mol Med Rep 14: 1187-1193, 2016.

17. Shen M, Tang W, Cao Z, Cao X and Ding F: Isolation of rat Schwann cells based on cell sorting. Mol Med Rep 16: 1747-1752, 2017.

18. Rodriguez Sanchez DN, de Lima Resende LA, Boff Araujo Pinto G, de Carvalho Bovolato AL, Possebon FS, Deffune E and Amorim RM: Canine adipose-derived mesenchymal stromal cells enhance neuroregeneration in a rat model of sciatic nerve crush injury. Cell Transplant 28: 47-54, 2019.

19. Liu H, Lv P, Wu H, Zhang K, Xu F, Zheng L and Zhao J: The proliferation enhancing effects of salidroside on schwann cells in vitro. Evid Based Complement Alternat Med 2017: 4673289, 2017.

20. Zhou W, Stukel JM, Cebull HL and Willits RK: Tuning the mechanical properties of poly(Ethylene Glycol) microgel-based scaffolds to increase 3D schwann cell proliferation. Macromol Biosci 16: 535-544, 2016.

21. Uz M, Buyukoz M, Sharma AD, Sakaguchi DS, Altinkaya SA and Mallapragada SK: Gelatin-based 3D conduits for transdifferentiation of mesenchymal stem cells into Schwann cell-like phenotypes. Acta Biomater 53: 293-306, 2017. 
22. Tabatabaei Qomi R and Sheykhhasan M: Adipose-derived stromal cell in regenerative medicine: A review. World J Stem Cells 9: 107-117, 2017.

23. Mazini L, Rochette L, Amine M and Malka G: Regenerative capacity of adipose derived stem cells (ADSCs), comparison with mesenchymal stem cells (MSCs). Int J Mol Sci 20: E2523, 2019.

24. di Summa PG, Kingham PJ, Raffoul W, Wiberg M, Terenghi G and Kalbermatten DF: Adipose-derived stem cells enhance peripheral nerve regeneration. J Plast Reconstr Aesthet Surg 63: 1544-1552, 2010.

25. Kolar MK and Kingham PJ: Regenerative effects of adipose-tissue-derived stem cells for treatment of peripheral nerve injuries. Biochem Soc Trans 42: 697-701, 2014.

26. Schilling BK, Schusterman MA II, Kim DY, Repko AJ, Klett KC, Christ GJ and Marra KG: Adipose-derived stem cells delay muscle atrophy after peripheral nerve injury in the rodent model. Muscle Nerve 59: 603-610, 2019.

27. Erba P, Mantovani C, Kalbermatten DF, Pierer G, Terenghi G and Kingham PJ: Regeneration potential and survival of transplanted undifferentiated adipose tissue-derived stem cells in peripheral nerve conduits. J Plast Reconstr Aesthet Surg 63: e811-e817, 2010

28. Georgiou M, Golding JP, Loughlin AJ, Kingham PJ and Phillips JB: Engineered neural tissue with aligned, differentiated adipose-derived stem cells promotes peripheral nerve regeneration across a critical sized defect in rat sciatic nerve. Biomaterials 37: 242-251, 2015.

29. Hei WH, Kim S, Park JC, Seo YK, Kim SM, Jahng JW and Lee JH: Schwann-like cells differentiated from human dental pulp stem cells combined with a pulsed electromagnetic field can improve peripheral nerve regeneration. Bioelectromagnetics 37: 163-174, 2016.

30. Bayat N, Ebrahimi-Barough S, Ardakan MM, Ai A, Kamyab A, Babaloo $\mathrm{H}$ and Ai J: Differentiation of human endometrial stem cells into schwann cells in fibrin hydrogel as 3D culture. Mol Neurobiol 53: 7170-7176, 2016.

31. Tohill M, Mantovani C, Wiberg M and Terenghi G: Rat bone marrow mesenchymal stem cells express glial markers and stimulate nerve regeneration. Neurosci Lett 362: 200-203, 2004.

32. Jessen KR and Mirsky R: The repair Schwann cell and its function in regenerating nerves. J Physiol 594: 3521-3531, 2016.

33. Dowsing BJ, Morrison WA, Nicola NA, Starkey GP, Bucci T and Kilpatrick TJ: Leukemia inhibitory factor is an autocrine survival factor for Schwann cells. J Neurochem 73: 96-104, 1999.

34. Meier C, Parmantier E, Brennan A, Mirsky R and Jessen KR Developing Schwann cells acquire the ability to survive without axons by establishing an autocrine circuit involving insulin-like growth factor, neurotrophin-3, and platelet-derived growth factor-BB. J Neurosci 19: 3847-3859, 1999.

35. Weiner JA and Chun J: Schwann cell survival mediated by the signaling phospholipid lysophosphatidic acid. Proc Natl Acad Sci USA 96: 5233-5238, 1999.

36. Liu Y, Zhang Z, Qin Y, Wu H, Lv Q, Chen X and Deng W: A new method for Schwann-like cell differentiation of adipose derived stem cells. Neurosci Lett 551: 79-83, 2013.

37. Zhang Y, Luo H, Zhang Z, Lu Y, Huang X, Yang L, Xu J, Yang W, Fan X, Du B, et al: A nerve graft constructed with xenogeneic acellular nerve matrix and autologous adipose-derived mesenchymal stem cells. Biomaterials 31: 5312-5324, 2010.

38. Luo H, Zhang Y, Zhang Z and Jin Y: The protection of MSCs from apoptosis in nerve regeneration by TGFbetal through reducing inflammation and promoting VEGF-dependent angiogenesis. Biomaterials 33: 4277-4287, 2012

39. Gu X, Ding F and Williams DF: Neural tissue engineering options for peripheral nerve regeneration. Biomaterials 35 6143-6156, 2014.

40. Wang Y, Li D, Wang G, Chen L, Chen J, Liu Z, Zhang Z, Shen H, Jin Y and Shen Z: The effect of co-transplantation of nerve fibroblasts and Schwann cells on peripheral nerve repair. Int J Biol Sci 13: 1507-1519, 2017

41. Cattin AL and Lloyd AC: The multicellular complexity of peripheral nerve regeneration. Curr Opin Neurobiol 39: 38-46, 2016.

42. Lehmann HC and Hoke A: Use of engineered Schwann cells in peripheral neuropathy: Hopes and hazards. Brain Res 1638: 97-104, 2016

43. La Noce M, Mele L, Tirino V, Paino F, De Rosa A, Naddeo P, Papagerakis P, Papaccio G and Desiderio V: Neural crest stem cell population in craniomaxillofacial development and tissue repair. Eur Cells Mater 28: 348-357, 2014.
44. Lee JK, Choi IS, Oh TI and Lee E: Cell-surface engineering for advanced cell therapy. Chemistry 24: 15725-15743, 2018.

45. Orbay H, Uysal AC, Hyakusoku H and Mizuno H: Differentiated and undifferentiated adipose-derived stem cells improve function in rats with peripheral nerve gaps. J Plast Reconstr Aesthet Surg 65: 657-664, 2012

46. Ladak A, Olson J, Tredget EE and Gordon T: Differentiation of mesenchymal stem cells to support peripheral nerve regeneration in a rat model. Exp Neurol 228: 242-252, 2011.

47. Cai S, Tsui YP, Tam KW, Shea GK, Chang RS, Ao Q, Shum DK and Chan YS: Directed differentiation of human bone marrow stromal cells to fate-committed schwann cells. Stem Cell Reports 9: 1097-1108, 2017.

48. Li X, Wang M, Jing X, Guo W, Hao C, Zhang Y, Gao S, Chen $\mathrm{M}$, Zhang $\mathrm{Z}$, Zhang $\mathrm{X}$, et al: Bone marrow- and adipose tissue-derived mesenchymal stem cells: Characterization, differentiation, and applications in cartilage tissue engineering. Crit Rev Eukaryot Gene Expr 28: 285-310, 2018.

49. Huang SJ, Fu RH, Shyu WC, Liu SP, Jong GP, Chiu YW, Wu HS, Tsou YA, Cheng CW and Lin SZ: Adipose-derived stem cells: Isolation, characterization, and differentiation potential. Cell Transplant 22: 701-709, 2013

50. Dubey NK, Mishra VK, Dubey R, Deng YH, Tsai FC and Deng WP: Revisiting the advances in isolation, characterization and secretome of adipose-derived stromal/stem cells. Int J Mol Sci 19: E2200, 2018.

51. Lei Z, Yongda L, Jun M, Yingyu S, Shaoju Z, Xinwen Z and Mingxue Z: Culture and neural differentiation of rat bone marrow mesenchymal stem cells in vitro. Cell Biol Int 31: 916-923, 2007.

52. Kingham PJ, Mantovani $C$ and Terenghi G: Notch independent signalling mediates Schwann cell-like differentiation of adipose derived stem cells. Neurosci Lett 467: 164-168, 2009.

53. Li X, Liao D, Gong P, Yuan Q and Tan Z: Neural differentiation of adipose-derived stem cells by indirect co-culture with Schwann cells. Arch Biol Sci 61: 703-711, 2009.

54. Jiang L, Jones S and Jia X: Stem cell transplantation for peripheral nerve regeneration: Current options and opportunities. Int J Mol Sci 18: E94, 2017

55. Pan Y and Cai S: Current state of the development of mesenchymal stem cells into clinically applicable Schwann cell transplants. Mol Cell Biochem 368: 127-135, 2012.

56. Yousefi F, Lavi Arab F, Nikkhah K, Amiri H and Mahmoudi M: Novel approaches using mesenchymal stem cells for curing peripheral nerve injuries. Life Sci 221: 99-108, 2019.

57. Sun AX, Prest TA, Fowler JR, Brick RM, Gloss KM, Li X, DeHart M, Shen H, Yang G, Brown BN, et al: Conduits harnessing spatially controlled cell-secreted neurotrophic factors improve peripheral nerve regeneration. Biomaterials 203: 86-95, 2019.

58. Cui Y, Yao Y, Zhao Y, Xiao Z, Cao Z, Han S, Li X, Huan Y, Pan J and Dai J: Functional collagen conduits combined with human mesenchymal stem cells promote regeneration after sciatic nerve transection in dogs. J Tissue Eng Regen Med 12: 1285-1296, 2018.

59. Kemp SW, Cederna PS and Midha R: Comparative outcome measures in peripheral regeneration studies. Exp Neurol 287: 348-357, 2017.

60. Zhang Z, Liu Y, Zhu X, Wei L, Zhu J, Shi K, Wang G and Pan L: Sciatic nerve leachate of cattle causes neuronal differentiation of PC12 cells via ERK1/2 signaling pathway. J Vet Sci 19: 512-518, 2018.

61. Shakhbazau A, Martinez JA, Xu QG, Kawasoe J, van Minnen J and Midha R: Evidence for a systemic regulation of neurotrophin synthesis in response to peripheral nerve injury. J Neurochem 122: 501-511, 2012.

62. Nurcombe V, Hill MA, Eagleson KL and Bennett MR: Motor neuron survival and neuritic extension from spinal cord explants induced by factors released from denervated muscle. Brain Res 291: 19-28, 1984.

63. Jessen KR, Mirsky R and Lloyd AC: Schwann cells: Development and role in nerve repair. Cold Spring Harb Perspect Biol 7 : a020487, 2015 .

This work is licensed under a Creative Commons Attribution-NonCommercial-NoDerivatives 4.0 International (CC BY-NC-ND 4.0) License. 